\title{
Técnicas de caracterización superficial. Situación y necesidades de la industria de dispositivos médicos en Costa Rica
}

\author{
Surface characterization techniques. Situation and needs of the \\ medical industry in Costa Rica
}

\author{
María Fernanda Luna Muñoz. \\ Universidad de Costa Rica \\ Escuela de Ingeniería Química \\ maria.lunamunoz@ucr.ac.cr \\ Lautaro Ramírez Varas \\ Universidad de Costa Rica \\ Escuela de Ingeniería Química \\ lautaro.ramirezvaras@ucr.ac.cr
}

\section{Resumen}

El presente documento tiene como objetivo identificar los procesos de caracterización química superficial de materiales, que necesita el mercado de la industria de dispositivos médicos, para evaluar el potencial mercado que podría tener éste en dicha industria.

Se realizaron entrevistas y visitas informativas a las empresas, para determinar sus necesidades en caracterizaciones químicas de la superficie. Esto será un primer abordaje para promover el encadenamiento productivo y la diversificación de los servicios que puede brindar el país.

Como resultado se obtiene que hay un gran desconocimiento técnico en el país relacionado al alcance de estas técnicas de caracterización superficial algunos de los problemas rutinarios, que las empresas expresaron tener, así como la innovación tecnológica podrían ser abordados si existiese el servicio en el país con las debidas acreditaciones para estos ensayos.

Palabras clave:

Caracterización superficial, dispositivos médicos, Espectroscopia Fotoelectrónica de Rayos X (XPS), encadenamientos productivos.

\footnotetext{
Abstract

The aim of this document is to identify process where chemical characterization of materials is needed in the medical device industry. Inquest and informative visits were applied to determine the industry needs on chemical surface characterization.
} 
This work is a first approach to promote the production linkages and the diversification of the laboratory services offered in Costa Rica.

The main result is that there is a lack of technical information related to the scope of these superficial characterization techniques. Some of the usual production problems expressed by the companies, as well as technological innovation could be addressed if the laboratory service in the country existed with the proper accreditations.

Keywords:

Surface characterization, medical devices, X-ray photoelectron spectroscopy (XPS), production linkages in Costa Rica. 


\section{INTRODUCCIÓN}

Durante la última década, Costa Rica ha sido partícipe de un constante crecimiento de la inversión extranjera directa (IED). Este se ha convertido en parte del modelo de desarrollo del sector de manufactura., permitiendo generar oportunidades de empleo, especialmente por el conglomerado de empresas de manufactura de dispositivos médicos. El conglomerado se ha instalado en el país bajo el régimen de zonas francas producen desde Costa Rica (entiéndase manufactura, suplidores e industria de dispositivos de capital nacional e internacional) dispositivos que son utilizados alrededor del mundo. El sector de ciencias de la vida es hoy uno de los más dinámicos en el país en términos de exportaciones y empleo. La llegada de empresas en este sector ha crecido nueve veces desde el año 2000 al 2015, pasando en el 2000 de 8 empresas del sector a 68 que se contabilizaron en el 2015. (CINDE, 2017) Además 6 de las 10 empresas de dispositivos cardiovasculares más grandes del mundo cuentan con plantas de operaciones en Costa Rica, (CINDE, 2016) siendo el segundo país exportador de dispositivos médicos en América Latina.(PROCOMER, Cinde, \& CR biomed, 2014)

Según cifras de PROCOMER 2016 (Promotora de Comercio Exterior de Costa Rica), el país exportó \$ 2500 millones en dispositivos médicos, representando el 26 $\%$ del total de las exportaciones de Costa Rica en este mismo año, casi igualando al sector agrícola. (Alpízar, 2017) Igualmente en términos de generación de empleo el sector ha crecido 13 veces su tamaño entre el año 2000 y el 2015, alcanzando más de 19 000 puestos de trabajo, según La Coalición Costarricense de Iniciativas de Desarrollo (CINDE. Se espera que las empresas de dispositivos médicos instaladas en Costa Rica sigan creciendo y evolucionen hacia productos más complejos, pasando de producir dispositivos Clase I a Clase II y Clase III (CINDE, 2017).

Aunque el país posee ventajas competitivas que permite la llegada de más empresas de este tipo, hay retos que pueden frenar el avance, ya que pese a los esfuerzos realizados para la consolidación de este capital extranjero, no se ha logrado tener un gran impacto en la transferencia tecnológica, la integración de los productores nacionales y los encadenamientos productivos han sido muy limitados. En un diagnóstico sobre el impacto que ha tenido la inversión extranjera directa en el país, Cordero y Paus señalan como la inversión extranjera directa (IED) no ha contribuido de manera significativa al avance de los activos de conocimiento del país. Esto porque el aporte se ha reducido principalmente al entrenamiento de trabajadores y a mejoras en los planes de estudio de las principales universidades.(Cordero \& Paus, 2008)

Dentro de los obstáculos que se presentan para fomentar los encadenamientos productivos y el desarrollo de una red de suministro local más extensa y de mayor profundidad que logre la inserción en las cadenas globales de valor están: la falta de capacitación de empresas locales, apoyo técnico y de financiamiento para los procesos de certificación y adquisición de equipo. (Govaere, 2014)

En el estudio internacional: "Cadenas Globales de Valor y diversificación de exportaciones: el caso de Costa Rica”, enumera desafíos para reforzar la competitividad interna: como promover encadenamientos entre empresas multinacionales y pequeñas y medianas empresas; aumentar recursos para invertir en innovación e investigación y desarrollo; adaptar oferta 
educativa a demandas del sector productivo; superar atrasos en infraestructura, logística y sector energético; ampliar la institucionalidad para la política de competitividad y lograr un mayor apoyo a la estrategia de inserción internacional. (Ramírez, 2014)

En el 2014, CINDE realizó un mapeo de las necesidades de laboratorio en las empresas de IED de manufactura avanzada y ciencias de la vida donde resaltan servicios de laboratorio que se requieren actualmente como lo son: laboratorios de calibración de patrones y equipos, laboratorios de análisis de materiales, composiciones químicas y características mecánicas, laboratorio de análisis químicos, laboratorios de mediciones ambientales y análisis de agua y laboratorios microbiológicos. Todos estos requieren acreditaciones (ISO 17025) del Ente Costarricense de Acreditación (ECA) trazables a patrones del National Institute of Standards and Technology NIST. («Mapeo necesidades de laboratorio», s. f.)

Es en este contexto que el trabajo desea mostrar un instrumento necesario para la innovación y la mejora de las capacidades de investigación. Lo anterior, a través del establecimiento de un laboratorio de análisis en química superficial que promueva un entorno más dinámico para el desarrollo científico y tecnológico del país. Este estudio pretende exponer un caso específico para colaborar con los esfuerzos realizados en el mapeo de las necesidades de CINDE. («Mapeo necesidades de laboratorio», s. f.)

Algunos ejemplos de aplicación de análisis superficial en la industria de dispositivos médicos se mencionan a continuación:

- La colonización de bacterias en dispositivos médicos corresponde a una de las complicaciones más graves después de la implantación. Para ayudar a inhibir este crecimiento en los dispositivos poliméricos, se desarrolló un recubrimiento antimicrobiano basado en guanidina, el cual se adhiere al cloruro de polivinilo (PVC). Para analizar la zona externa del PVC recubierto y observar el comportamiento del compuesto desarrollado en diferentes situaciones de contaminación, los expertos han utilizado la espectroscopía fotoelectrónica de rayos X (XPS). (Villanueva, González, Rodríguez-Castellón, Teves, \& Copello, 2016)

- El polimetilmetacrilato, se utiliza para rellenar el espacio entre una prótesis y su hueso circundante en artroplastias cementadas; se sabe que los problemas de biocompatibilidad relacionados con la aplicación de cemento óseo limitan el éxito clínico de estos dispositivos. Por lo tanto hay estudios que utilizan XPS para saber si hay una correlación entre los cambios de superficie y el tiempo de envejecimiento. (Brittain, 2006)

- Para entender el origen las fallas adhesivas a menudo se requieren análisis sofisticados. La ubicación de la falla puede estar en una capa muy delgada en una interfaz. La interfaz puede haberse contaminado durante cualquiera de los procesos utilizados para preparar la superficie de adherencia o después. Un ejemplo clásico en el que un agente de liberación de silicona o dimetil siloxano o lubricante causó fallas en la interfaz de fritas de vidrio. Esta capa delgada de dimetil siloxano no fue detectada por dos investigaciones previas de laboratorio, pero fue detectada fácilmente en ests laboratorio usando el análisis de superficie XPS. (Anderson Materials Evaluation Inc, 2015)

- La solución de problemas de contaminación superficial a menudo requiere el conocimiento del estado químico del contaminante. Como lo es el caso en la detección 
de cloro en la superficie, el análisis de la información del fotoelectrón revela si el cloro está presente en forma de ácido clorhídrico o triclorobenceno. El conocimiento de la forma del cloro acelera la localización y eliminación de la fuente de contaminación del proceso. (Van Zant, 2014)

El problema puntual a ser estudiado radica mapear si existen algunas de éstas problemáticas usuales en las empresas instaladas en Costa Rica, las cuáles son solventadas con técnicas de caracterización superficial (ensayos acreditados). Actualmente estos ensayos no se realizan en el país. Se escogió previamente esta necesidad debido a su impacto en la fabricación y la caracterización de materiales.

\section{INFRAESTRUCTURA}

El Estado de la Nación se refiere al estado de la infraestructura de las unidades de investigación y desarrollo (I\&D) como "el potencial innovador de un país". Ésta es definida como una variable crítica en la medida en que potencia o limita la capacidad de generar conocimiento y favorecer la vinculación entre los distintos actores para la ciencia, la tecnología y la innovación. El estado y tipo de equipamiento, las prácticas de colaboración entre estos centros y los sectores productivos, proporcionan los medios para que el personal involucrado en actividades de I\&D pueda desarrollar nuevos conocimientos y aplicaciones que, eventualmente, serán transferidos y usados en los procesos productivos. (Estado de la Nación, 2014)

Sobre los centros de investigación (CI), PROCOMER los resalta como un actor importante dentro de las industrias de alta tecnología. Y resalta que los CI que son partes de las universidades públicas de Costa Rica; no solo cumplen con un objetivo educativo, sino que también proporcionan servicios importantes a las empresas del sector privado y actúan como apoyo para el desarrollo de nuevos productos. (PROCOMER et al., 2014)

El $80 \%$ de la investigación de las ciencias de la vida es realizado por universidades públicas, ya que las unidades de I+D están concentradas en el sector académico de estas instituciones. (PROCOMER et al., 2014)

\subsection{Capacidades Nacionales en la caracterización de Materiales}

Las universidades estatales en Costa Rica tienen centros de investigación (CI) que se dedican al análisis y caracterización de materiales en diversas áreas y sectores como lo son:

- CICIMA (El Centro de Investigación en Ciencia e Ingeniería de Materiales) de la Universidad de Costa Rica;

- LANOTEC (Laboratorio Nacional de Nanotecnología) del Centro Nacional de Alta Tecnología;

- CIEMTEC (El Centro de Investigación y Extensión en Materiales) del Tecnológico de Costa Rica;

- $\quad$ LAMI (El Laboratorio de Materiales Industriales) de la Universidad Nacional de Costa Rica. 
- CICANUM (Centro de Investigación en Ciencias Atómicas, Nucleares y Moleculares)

Los centros de investigación cuentan con diferentes equipos y técnicas especializadas (técnicas no acreditadas con el ECA o no trazables a materiales de referencia certificados), de todas las técnicas que realizan ninguna es de caracterización química superficial.

Ofertas técnicas de caracterización por parte de los centros de investigación:

- Microscopía electrónica de barrido (SEM)

- Microscopía electrónica de transmisión (TEM)

- Espectroscopía de rayos X dispersados (EDX)

- Difractometría de rayos X (XRD)

- Espectrómetro infrarrojo por transformada de Fourier con aditamento para reflexión total interna (FTIR- ATR)

- Espectroscopia RAMAN, UV, luz visible.

- Microscopía de Fuerza atómica AFM.

A pesar de esta gran cantidad de técnicas en caracterización de materiales que hay en el país, éste no cuenta con ninguna técnica de caracterización superficial acreditada y complementaria a las ya existentes en Costa Rica. (CIEMTEC, 2017), (CICIMA, 2017), (LANOTEC, 2017), (LAMI, 2017), (CICANUM, 2017).

\section{Normativa}

La industria de los dispositivos médicos es uno de los sectores más regulados. Se deben satisfacer sistemas de calidad y requerimientos de producto significativos para asegurar que los dispositivos producidos sean adecuados para su propósito. De ahí la gran importancia de las acreditaciones, ya que son la respuesta a las crecientes exigencias de garantías requeridas por el mercado, tanto a nivel de la industria como del consumidor final. Los organismos de acreditación, como el Ente Costarricense de Acreditación (ECA), desempeñan su tarea conforme a los mismos criterios internacionales, utilizando métodos de evaluación equivalentes y transparentes. La acreditación, garantiza que los OEC (Laboratorios de ensayos, calibración, unidades de inspección y organismos de certificación), de distintos países desempeñan su tarea de manera equivalente, generando la adecuada confianza que posibilita la aceptación mutua de resultados.(Ente Costarricense de Acreditación, 2017)

Dentro de las normas internacionales de certificación que requieren las organizaciones involucradas en una o más etapas del ciclo de la vida de los dispositivos médicos están:

- ISO-13485: especifica los requisitos para un Sistema de gestión de la calidad (SGC), en el que una organización necesita demostrar su capacidad para proporcionar dispositivos médicos y servicios relacionados que cumplan consistentemente con el cliente y los requisitos reglamentarios aplicables. (Internacional Organization of Standardizacion, 2017)

- ISO/IEC 17025:2005: especifica los requisitos generales para la competencia de laboratorios de ensayo o calibración, cubre las pruebas y la calibración realizadas 
utilizando métodos estándar, métodos no estándar y métodos desarrollados en el laboratorio. (Internacional Organization of Standardizacion, 2017)

\section{ESPECTROSCOPIA FOTOELECTRÓNICA DE RAYOS X (XPS)/ TÉCNICA DE CARACTERIZACIÓN SUPERFICIAL}

También conocida como Espectroscopía Electrónica para Análisis Químico (ESCA), es una herramienta espectroscópica no destructiva para estudiar superficies. Se puede estudiar cualquier material sólido y todos los elementos pueden detectarse mediante esta técnica (excepto el hidrógeno y el helio), generalmente con una abundancia de 0,1 por ciento atómico. (Patnaik \& Dean, 2004) La popularidad de la técnica radica en la alta sensibilidad superficial que alcanza, con profundidades de análisis de aproximadamente 3nm -10 nm. (Anderson Materials Evaluation Inc, 2015)

La Espectroscopia Fotoelectrónica de Rayos X (XPS), es uno de los métodos de caracterización de superficies ampliamente utilizado hoy en día. Su popularidad radica del alto contenido de información que suministra y la flexibilidad para ser utilizada en una gran variedad de muestras. La técnica XPS proporciona información química que los métodos clásicos no brindan, esta es utilizada en investigación, desarrollo de nuevos materiales y en controles de calidad en fabricación

\subsection{Principio de funcionamiento de XPS}

Las muestras se bombardean con un haz de rayos X de energía $h v$ (las dos fuentes más utilizadas son las líneas K $\alpha$ de magnesio 1254 eV, y aluminio 1487 eV) y de ella se desprenden electrones del interior con una energía cinética $E_{k}$ que supera la energía de enlace $E_{B}$ y la función de trabajo $(\varnothing)$. Estos electrones interiores se llaman fotoelectrones de rayos $\mathrm{X}$. La ecuación de la energía puede expresarse de la manera siguiente:

$$
E_{k}=h v-E_{B}+\emptyset
$$

Donde:

$E_{k}=$ Energía cinética, $\mathrm{eV}$

$E_{B}=$ Energía de enlace, $\mathrm{eV}$

$(\varnothing)=$ Fuerza de trabajo, eV

$h v=$ energía del haz de rayos $\mathrm{X}, \mathrm{eV}$

Las energías cinéticas de estos electrones expulsados que se originan en los primeros $30 \AA ̊$ de la superficie de la muestra se miden mediante la XPS. (Seymour \& Carraher, 2002)

La utilidad de determinar la $E_{B}$ es porque la energía de una capa electrónica en un átomo particular es característica de ese átomo. Por lo tanto, determinando $E_{B}$ a través de la medición de la $\mathrm{E}_{\mathrm{k}}$, se identifica qué átomo está involucrado en el proceso. (Brundle, Watts, \& Wolstenholme, 2004) 


\subsection{Aplicaciones de la técnica y campo de estudio}

Es la única técnica (junto con la Espectroscopia Auger para luz Síncrotron) comercial que permite determinar los compuestos químicos que se encuentran en la superficie de los materiales. Es posible, así obtener información de las primeras capas (del orden de los nanómetros) de la superficie del material, obviando la profundidad de este. A diferencia de otros métodos como el SEM (microscopía electrónica de barrido) y el EDX (espectroscopia de energía dispersiva) que brindan información de toda la profundidad del material, como se muestra en la tabla 1.

Con el XPS, se pueden obtener análisis elementales, información sobre los estados de oxidación, grupos funcionales orgánicos, análisis cuantitativos, sea como porcentajes de los elementos o razones de estados de oxidación, distribuciones de materiales a través de la superficie, también determinar si el material está presente como una película delgada en la superficie (grosor de la película, uniformidad en el grosor y composición química de la película). Dentro de los campos de estudio del XPS están: películas delgadas y recubrimientos, polímeros y adhesivos, contaminación y limpieza de superficies, funcionalización de superficies, corrosión, microelectrónica y semiconductores, caracterización de superficies sólidas y catálisis. (Anderson Materials Evaluation Inc, 2015)

Tabla 1. Resumen de las técnicas de caracterización de materiales más utilizadas.

\begin{tabular}{lcl}
\hline \multicolumn{1}{c}{ Técnica } & $\begin{array}{l}\text { Acrónimo } \\
\text { en Inglés }\end{array}$ & \multicolumn{1}{c}{ Información obtenida } \\
\hline $\begin{array}{l}\text { Espectroscopía } \\
\text { Fotoelectrónica de rayos X }\end{array}$ & XPS & $\begin{array}{l}\text { Identificación cualitativa y cuantitativa de todos los } \\
\text { elementos con alta sensibilidad superficial (incluye estados } \\
\text { de oxidación) que alcanza profundidades de análisis de } \\
\text { aproximadamente 3nm -10 nm. (Patnaik \& Dean, 2004) }\end{array}$ \\
$\begin{array}{l}\text { Microscopía electrónica de } \\
\text { barrido }\end{array}$ & SEM & $\begin{array}{l}\text { Técnica de imagen, caracterización de la morfología, } \\
\text { determina la estructura y tamaño de nanopartículas. } \\
\text { (Wiesner, 2016) }\end{array}$ \\
$\begin{array}{l}\text { Microscopía electrónica de } \\
\text { transmisión }\end{array}$ & TEM & $\begin{array}{l}\text { Proporciona imágenes estructurales con resolución a } \\
\text { nivel atómico. Tamaño y distribución de nano partículas. } \\
\text { (Wiesner, 2016) }\end{array}$ \\
$\begin{array}{l}\text { Espectroscopía de energía } \\
\text { dispersada }\end{array}$ & EDX & $\begin{array}{l}\text { Determinación de la composición elemental de todo el } \\
\text { bulk del material (1000 nm - 2000 nm). (Khandpur, 2013) }\end{array}$ \\
Difracción de rayos X & XRD & $\begin{array}{l}\text { Determinación de tamaño de partículas y estructuras } \\
\text { cristalinas, identificación de fases presentes. (Roque- } \\
\text { Malherbe, 2009) }\end{array}$ \\
\hline
\end{tabular}




\section{METODOLOGÍA PARA DETERMINAR NECESIDADES DE LA INDUSTRIA MÉDICA EN COSTA RICA}

\section{METODOLOGÍA}

\section{Parte preliminar}

PROCOMER, colaboró con la realización de encuestas a empresas de dispositivos médicos, de las cuáles obtuvo 8 respuestas.

\section{Posteriormente}

Se inscribió el proyecto de investigación en Auge (Agencia Universitaria para la gestión del Emprendimiento de la Universidad de Costa Rica), para obtener retroalimentación de cómo abordar la realización de entrevistas a otras empresas de dispositivos médicos.

Se eligió:

- Ingenieros de calidad o materiales de empresas que producen dispositivos médicos Clase II y Clase III. *

- Investigadores involucrados en caracterización de materiales de las universidades estatales.

- A modo exploratorio se entrevistó una empresa del sector aeroespacial y una del área de los semiconductores.

\section{Obtención de los insumos para la investigación}

Se realizó entrevistas personales, cuestionarios en línea y charlas informativas de la técnica XPS.

\section{Obtención de resultados}

Se obtuvo resultados cualitativos y cuantitativos. 
Para la obtención de resultados preliminares, se tuvo un primer acercamiento con la agencia Promotora de Comercio Exterior de Costa Rica (PROCOMER), la cual colaboró con la realización de un primer cuestionario con el cuál se obtuvo respuesta de 8 empresas del sector ciencias de la vida. Después se inscribió el proyecto de investigación en Auge (Agencia Universitaria para la gestión del Emprendimiento de la Universidad de Costa Rica), para obtener retroalimentación de cómo abordar la realización de entrevistas a otras empresas de dispositivos médicos, con un enfoque técnico.

Posteriormente se eligieron cuáles empresas de dispositivos médicos eran aptas para la investigación, con lo que se escogieron empresas que producen dispositivos médicos Clase II (dispositivos médicos de riesgo moderado y alto, sujetos a controles especiales en la fase de fabricación para demostrar su seguridad y efectividad) y Clase III (dispositivos médicos de muy alto riesgo sujetos a controles especiales, destinados a proteger o mantener la vida o para un uso de importancia sustancial en la prevención del deterioro de la salud humana, o si su uso presenta un riesgo potencial de enfermedad o lesión) (INVIMA, 2013). Se eligió una muestra de 10 empresas de dispositivos médicos, dentro de las cuáles se encuentran algunas de las más grandes y posicionadas dentro del clúster, así como las que fabrican dispositivos de alta complejidad.

También se consideró relevante entrevistar a tres investigadores uno del LANOTEC, uno del CICIMA, y otro del LAMI, involucrados en caracterización de materiales de las universidades estatales, adicionalmente a modo exploratorio se entrevistó a una empresa del sector aeroespacial y otra del sector de semiconductores.

Se enviaron cuestionarios vía correos electrónicos y se visitó empresas con charlas informativas. Estas charlas tenían un enfoque en espectroscopía fotoelectrónica de rayos $\mathrm{X}$, para determinar la situación y necesidades de cada empresa en específico en cuánto a técnicas de caracterización superficial con lo que se obtuvo resultados tanto cuantitativos como cualitativos.

Debido al desconocimiento que se identificó en las encuestas de la etapa preliminar acerca de las técnicas de caracterización superficial, se procedió en las siguientes encuestas y entrevistas a dar ejemplos de problemas comunes que solo pueden ser resueltos con el uso de la técnica de espectroscopia fotoelectrónica de rayos X (explicados en la introducción), para identificar el posible uso potencial de la técnica como variable de estudio. Posteriormente se preguntó cómo solventaban éstos problemas.

Para la codificación de las encuestas y entrevistas, se buscó hilos conectivos y patrones entre las distintas categorías que pudieran llamarse temas, ya que la mayoría de las respuestas son cualitativas. (Martin, 2013)

\section{RESULTADOS}

A las empresas, se les preguntó en inicialmente si realizan en Costa Rica o en el extranjero pruebas de caracterización de materiales (superficial) y cuál es la frecuencia de uso. Como se puede observar en la Figura 1,50 \% dijo que sí y el otro $50 \%$ dijo que no. Respecto a la frecuencia de uso, respondieron que depende del día a día, ya que pueden ser desde mensuales hasta anuales. Igualmente al indicar cuál son las pruebas más comunes 
que realizan destacaron pruebas no superficiales: Espectroscopía de energía dispersada (EDX), Microscopía electrónica de barrido (SEM), Espectroscopía Infrarroja por Transformada de Fourier (FTIR), Termogravimetría (TGA), Ángulo de contacto y Microscopía electrónica de transmisión (TEM). A lo que podemos observar que ninguna de las empresas nombró la Espectroscopía Fotoelectrónica de rayos X (XPS). Denotando la carencia de la técnica en el total de la población de estudio y el desconocimiento, de la gran mayoría sobre el significado de las técnicas de caracterización superficial.

Adicionalmente, se menciona que en reiteradas ocasiones para resolver problemas de caracterización de materiales deben incurrir en el gasto para enviar muestras a laboratorios en el extranjero o a sus respectivas casas matrices. Esto porque en Costa Rica no es posible suplir esa necesidad; sea por problemas de disponibilidad, logística o certificaciones.

\section{Caso centro de investigación}

En el caso de los investigadores de las universidades, éstos indican que debido a los recursos limitados en estos centros, deben limitar las investigaciones a los equipos con que se cuenta a nivel local.

Porcentaje de uso de técnicas de caracterización superficial de materiales en la industria de dispositivos médicos

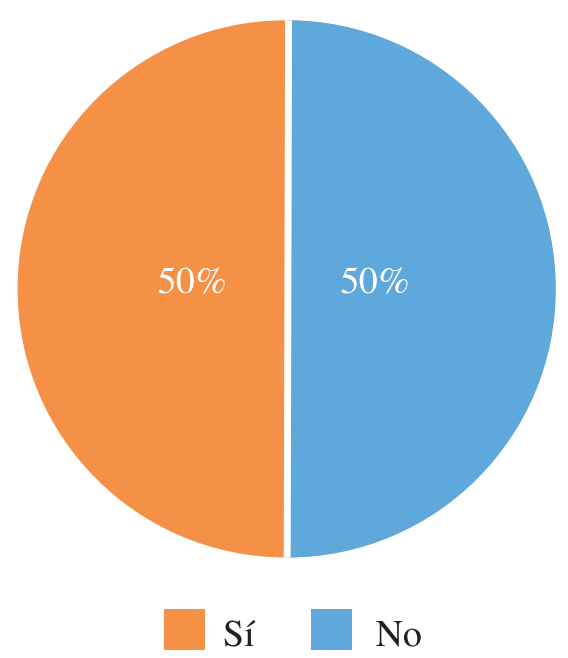

Figura 2 Porcentaje de uso de técnicas de caracterización superficial de materiales en la industria de dispositivos médicos.

En la Figura 3, se puede observar como en las empresas de dispositivos médicos y en la aeroespacial entrevistadas, existe un gran desconocimiento de la funcionalidad de la técnica de caracterización superficial XPS, ya que solo un $36 \%$ de las empresas 
entrevistadas habían escuchado hablar de la técnica, y aun así este pequeño porcentaje no conoce a profundidad las ventajas y los alcances de la técnica.

\section{Situación actual de la demanda en caracterización superficial.}

Posterior a la etapa preliminar, las empresas identificaron la aplicabilidad de utilizar XPS en sus procesos y una alternativa complementaria de cómo resolver problemas que, muchas veces no saben cómo erradicarlos adecuadamente. Como consecuencia generaron una lista de problemas a los que se enfrentan con frecuencia:

- Determinar si corrosiones son por subproductos o procesamiento del material.

- Determinar residuos en la superficie de dispositivos después de aplicar materiales de limpieza.

- Analizar contaminantes superficiales que no son capturados por el EDX.

- Descartar si manchas en materiales son subproductos o no del proceso.

- Análisis causa raíz sobre el debonding entre dos superficies.

- Análisis de pasivación de metales.

- Análisis y desarrollo de recubrimientos.

- Problemas en soldaduras.

- Desarrollo de materiales de alta conductividad, semiconductores.

Conocimiento de las empresas de dispositivos médicos acerca de la Espectroscopía Fotoelectrónica de rayos $\mathrm{X}$ (XPS)
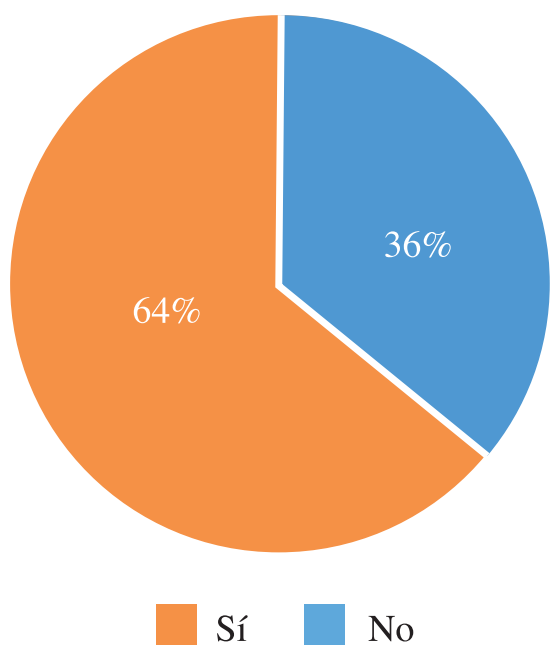

Figura 3. Conocimiento de las empresas de dispositivos médicos acerca de Espectroscopía Fotoelectrónica de rayos $\mathrm{X}$ (XPS). 
En el caso de las entrevistas realizadas a los investigadores, estos indican que el uso de XPS ayudaría a complementar los estudios que realizan en materiales y con esto incrementar la validez de las investigaciones, así como proyectar a Costa Rica en la punta de la investigación.

Después de identificar y conocer gran cantidad de casos en los que es de utilidad la técnica, el $72 \%$ de las empresas respondieron que estarían interesados en utilizarla, como se muestra en la Figura 4. Sin embargo consideran que se deben dar más charlas acerca del funcionamiento de la técnica para crear el conocimiento en el recurso humano de las empresas.

También se hace énfasis en que en la industria de dispositivos médicos al ser una de las más reguladas del mundo, debe solicitar que los laboratorios de venta de servicios cuenten con equipos trazables a patrones internacionales y en la mayoría de los casos también deben contar con las certificaciones correspondientes al ciclo de la vida de los dispositivos médicos.

Interés de las empresas de dispositivos

médicos acerca de la Espectroscopía Fotoelectrónica

de rayos X (XPS)

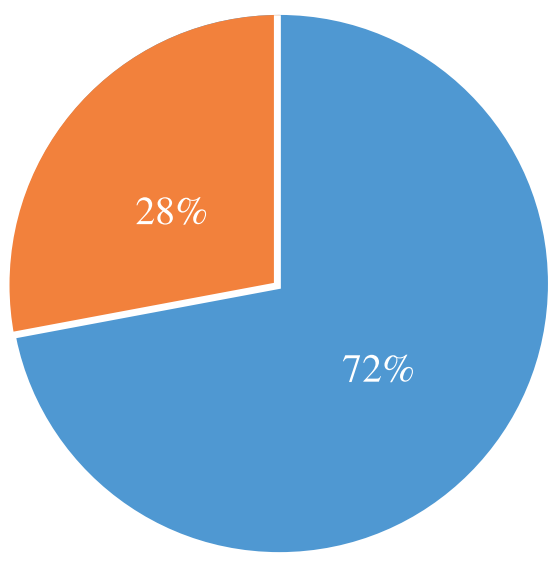

Sí No

Figura 4. Interés de las empresas de dispositivos médicos en utilizar Espectroscopía Fotoelectrónica de rayos X (XPS).

\section{Facilidades deseables por la industria dentro del país}

Para finalizar las entrevistas se les consultó adicionalmente sobre que facilidades consideran necesita Costa Rica para potenciar el área de investigación y desarrollo y venta de servicios en el campo de materiales, algunas de las respuestas más comunes fueron:

- Asignar más fondos a los centros de investigación para realizar Investigación y Desarrollo. 
- Fomentar el vínculo industria- academia, para que las universidades se enfoquen en atender las necesidades industriales.

- Ofrecer cartera de servicios dentro de la ISO 17025 con cortos tiempos de respuesta.

- Aumentar el roce internacional con tecnologías de punta.

- Disminuir los impuestos para la obtención de equipos.

- Elevados tiempos de espera y problemas para sacar equipos de aduanas.

\section{CONCLUSIONES}

- Existe desconocimiento sobre las técnicas de caracterización química superficial, aunque éstas son necesarias en la resolución de problemas para la mayoría de las empresas encuestadas. No obstante, no hay evidencia que justifique la necesidad en frecuencia de uso por una sola empresa. Estos análisis actualmente se realizan en el exterior.

- Costa Rica debe contar con una estrategia que promueva la incorporación de más tecnología y valor agregado, en la cual se conozcan las necesidades del sector productivo. Y se integren proyectos de investigación que cuenten con la participación de la academia, sector público y privado.

- Se necesita más ayuda estatal para la consolidación de la investigación o emprendimientos de base tecnológica.

- El desarrollo de nuevos materiales, la investigación y controles de calidad en fabricación se ven limitados en Costa Rica, y no logran suplir las necesidades del sector productivo debido a la falta de equipo.

- Dentro de las limitaciones del estudio, se encuentra que no se logró entrevistar a la totalidad de la muestra, ya que no todas las empresas de dispositivos médicos estuvieron abiertos a contestar las entrevistas.

\section{DESAFÍOS}

- Estudiar el impacto de estas técnicas dentro del sector aeroespacial como otro aliado importante para lograr transferencia tecnológica e incursionar en nuevos mercados de alto valor tecnológico, lo que implica que los clientes están dispuestos a pagar más por productos que estén controlados y certificados. (sofisticación de los procesos)

- Fomentar en Costa Rica la apertura de un Laboratorio Nacional en Química de Superficie que cuente con las acreditaciones necesarias para dar soporte a la industria de dispositivos médicos, así como a la industria aeroespacial que cada vez se encuentra en mayor auge.

- Promover la venta de servicios por parte de los centros de investigación de las Universidades Estatales a las industrias de dispositivos médicos, para promover la transferencia tecnológica y aumentar los activos de conocimiento del país, así 
como discutir la pertinencia de la acreditación de los laboratorios de los centros de investigación por las limitaciones que crea a los fines académicos con los que fueron adquiridos los equipos.

\section{REFERENCIAS}

Alpízar, M. (2017). Estadísticas de comercio exterior Costa Rica 2016. PROCOMER. Recuperado a partir de http://procomer.com/downloads/estudios/estudio_ estadistico_2016/AnuarioEstadistico2016.pdf

Anderson Materials Evaluation Inc. (2015). Surface Analysis by X-Ray Photoelectron Spectroscopy - XPS |Anderson Materials Evaluation, Inc. Recuperado 5 de noviembre de 2017, a partir de http://www.andersonmaterials.com/xps.html

Brittain, H. (2006). X-Ray Photoelectron and X-Ray Fluorescence Spectroscopy. En H. Brittain \& H. Brittain, Spectroscopy of Pharmaceutical Solids (pp. 67-89). CRC Press. https://doi.org/10.1201/9780849361333.ch3

Brundle, C. R., Watts, J. F., \& Wolstenholme, J. (2004). X-ray photoelectron and Auger electron spectroscopy. Analytical Instrumentation Handbook, 399.

CICANUM. (2017). Unidad de Investigación - Centro de Investigación en Ciencias Atómicas, Nucleares y Moleculares (CICANUM). Recuperado 7 de noviembre de 2017, a partir de https://vinv.ucr.ac.cr/es/unidades/ centro-de-investigacion-en-ciencias-atomicas-nucleares-y-moleculares-cicanum

CICIMA. (2017). Equipos CICIMA. Recuperado 4 de noviembre de 2017, a partir de http://www.cicima.ucr.ac.cr/equipos

CIEMTEC. (2017). Centro de Investigación y Extensión de Ingeniería de los Materiales (CIEMTEC). Recuperado 4 de noviembre de 2017, a partir de https://www.tec.ac.cr/ centros-investigacion/centro-investigacion-extension-ingenieria-materiales-ciemtec/ servicios

Cordero, J., \& Paus, E. (2008). Foreign investment and economic development in Costa Rica: the unrealized potential. JSTOR.

Ente Costarricense de Acreditación. (2017). ECA -- ¿qué es la acreditación? Recuperado 4 de noviembre de 2017, a partir de http://www.eca.or.cr/acr_quees.php

Estado de la Nación. (2014). Estado de la ciencia, la tecnología y la innovación. Recuperado a partir de https://www.estadonacion.or.cr/ecti/assets/estado-cytpregunta-17.pdf

Govaere, V. (2014). En camino de una política de encadenamientos productivos en Costa Rica. OCEX-UNED. Recuperado a partir de https://www.uned.ac.cr/ocex/images/ stories/Hacia\%20una\%20poltica\%20de\%20encadenamientos\%20productivos\%20 en\%20CR.pdf

Internacional Organization of Standardizacion. (2017). ISO 13485:2016 - Medical devices -- Quality management systems -- Requirements for regulatory purposes. Recuperado 4 de noviembre de 2017, a partir de https://www.iso.org/standard/59752.html 
INVIMA. (2013). ABC de dispositivos médicos. Recuperado a partir de https://www. invima.gov.co/images/pdf/tecnovigilancia/ABC\%20Dispositivos $\% 20 \mathrm{Medicos} \% 20$ INVIMA.pdf

Khandpur. (2013). Handbook of Analytical Instruments. Maidenhead: McGraw-Hill Education. Recuperado a partir de http://accessengineeringlibrary.com/browse/ handbook-of-analytical-instruments-second-edition

LAMI. (2017). Servicios Laboratorio de Materiales Industriales. Recuperado 4 de noviembre de 2017, a partir de http://www.lami.una.ac.cr/index.php/es/servicios

LANOTEC. (2017). LANOTEC - CeNAT. Recuperado 4 de noviembre de 2017, a partir de http://www.cenat.ac.cr/es/lanotec/

Mapeo necesidades de laboratorio. (s. f.).

Martin, P. (2013). La ciencia de la investigación cualitativa. Ediciones UniandesUniversidad de los Andes.

Patnaik, P., \& Dean, J. A. (2004). Dean's analytical chemistry handbook (2nd ed). New York: McGraw-Hill.

PROCOMER, Cinde, \& CR biomed. (2014). Guía del Sector Ciencias de la Vida. Recuperado a partir de http://esencialcostarica.com/procomer/ebook/procomer_biotecnologia/ es/pdf/procomer_biotecnologia.pdf

Ramírez, K. (2014). Costa Rica: hacia una política de encadenamientos productivos. Recuperado a partir de https://www.uned.ac.cr/acontecer/a-diario/gestionuniversitaria/2081-costa-rica-hacia-una-politica-de-encadenamientos-productivos\#

Roque-Malherbe, R. (2009). The Physical Chemistry of Materials: Energy and Environmental Applications. CRC Press. https://doi.org/10.1201/9781420082739

Seymour, R. B., \& Carraher, C. E. (2002). Introducción a la química de los polímeros. Barcelona; México: Reverté.

Van Zant, P. (2014). Microchip fabrication: a practical guide to semiconductor processing (Sixth edition). New York: McGraw-Hill Professional.

Villanueva, M. E., González, J. A., Rodríguez-Castellón, E., Teves, S., \& Copello, G. J. (2016). Antimicrobial surface functionalization of PVC by a guanidine based antimicrobial polymer. Materials Science and Engineering: C, 67, 214-220. https:// doi.org/10.1016/j.msec.2016.05.052

Wiesner, M. R. (2016). Environmental nanotechnology, (2nd edition). New York, NY: McGraw-Hill Education. 